\title{
The Effect of Bending Load for Two Geometries of AISI 316L Cardiovascular Stent
}

\section{Benhaddou $\mathbf{M}^{\star}$ and Ghammouri $\mathbf{M}$}

ENSA, Équipe de Mécanique et Calcul Scientifique, Université Mohamed 1er, BP 696, Oujda, Morocco

*Corresponding author: Benhaddou M, ENSA, Mechanics and Scientific Computing team, Universite Mohamed 1er, BP 696, Oujda, Morocco, Tel: +212 624515348; Email: med.benhaddou88@gmail.com

Received date: March 14, 2018; Accepted date: April 04, 2018; Published date: April 09, 2018

Copyright: ( 92018 Benhaddou M. This is an open-access article distributed under the terms of the creative commons attribution license, which permits unrestricted use, distribution and reproduction in any medium, provided the original author and source are credited.

\begin{abstract}
In the present study, Abaqus finite element modelling was used to explore the durability of cardiovascular stent made of AISI 316L under bending loading. In order to achieve this purpose, two geometries of stent are explored: the U-shaped and $\Omega$-shaped stent. The cyclic loading of blood pressure doesn't cause fracture. This result was confirmed by many studies. However, when subjected to bending loading the stent was found to experience an inservice failure. The last proved to the artery movement and depends on the increase of bending loading. However, the $\Omega$-shape stunt permits a better distribution of load than the U-shape one.
\end{abstract}

Keywords: Abaqus numerical simulation; Bending; Stent; Effect of design

\section{Introduction}

Cardiovascular stents are medical devices inserted into the artery to restore blood flow and keep the blood vessel open. Although the design of stents is a relatively old subject, there are numerous studies on stent rupture that may be due to restenosis [1-3] or fatigue [4,5]. Another study deals with the new generation of drug-eluting stents that have been developed to prevent restenosis [6], despite this development several studies show the deformation and stent rupture $[7,8]$. This break requires a better understanding of the stent behavior and the development of the tools for stent rupture. The stent deployment consists in introducing a small expandable balloon at the angioplasty site through a small puncture. The stent is then expanded via the inflation of the balloon inside it. After deflation and extraction of the balloon, the stent undergoes a plastic deformation after recoil in some critical zones which may be very harmful for its integrity.

The stent used in this study is made of stainless steel AISI 316L the choice of this material is based on its resistance to both the large amount of deformation caused by the balloon swelling $[9,10]$ and long- term loading cyclic resulting from arterial pressure [11], in addition to its biocompatibility and its resistance to corrosion. The deployment of the stent depends on the balloon inflation pressure, many cardiologists deploy the stents to a diameter greater than that required to ensure adequate permeability of the vessel to account for the elastic recoil of the device [12]. In the general case the stents must withstand at least 10 years of service this important duration requires very expensive experimental tests and a lot of time even at an accelerated frequency. For this reason, numerical simulation is a useful tool for predicting stent behavior and providing the most appropriate design [13].

The present work describes the influence of bendings on the durability of AISI 316L stents via a finite element based analysis using Abaqus software [9]. For this goal, a preliminary study consisting of the deployment of the stent is first performed using Abaqus explicit. Finally, the effect of bending loading on the stent resistance is pointedout.

\section{Materials}

The present work focuses on $316 \mathrm{~L}$ stainless steel with chemical composition and mechanical properties given in Table 1 and 2.

\begin{tabular}{|l|l|l|l|l|l|l|l|l|l|}
\hline $\begin{array}{l}\text { Composition } \\
\text { AISI 316L }\end{array}$ & $\mathbf{C}$ & Cr & Ni & Mo & Si & Mn & P & S & N \\
\cline { 2 - 8 } & 0.015 & 16.5 & 10.10 & 2 & 0.46 & 1.63 & 0.032 & 0.027 & 0.026 \\
\hline
\end{tabular}

Table 1: Chemical composition for AISI 316L

\begin{tabular}{|l|l|l|l|}
\hline Tensile strength Rm (MPa) & Yield stress $\sigma \mathbf{y}(\mathbf{M P a})$ & Strain to failure $\mathbf{\varepsilon f}(\%)$ & Young's modulus E (GPa) \\
\hline 580 & 315 & 37 & 210 \\
\hline
\end{tabular}

Table 2: Mechanical properties of 316L steel. 


\section{Mesh}

The numerical simulation of the mechanical stent behaviour is composed of two steps. The first consists of the stent deployment. The second deals with the application of bending loading. For a good accuracy of the results, an optimal meshing is needed prior to numerical computation. The meshing criterion is based on the combination of the time and results. Among solid elements, linear hexahedrons one is found to be the best candidate for stent models. As stents are loaded dominantly in bending, the fully-integrated linear hexahedron element (C3D8) is not suited for stent analysis. We recommend choosing the reduced-integration (C3D8I). Indeed, this type of elements does not suffer from hourglassing and has integration points closer to the surface. To optimise the finite element mesh, a convergence study with varying mesh density was performed until a balance between solution accuracy and efficiency is obtained. In this regard, four refinement levels were studied. Based on the results for the peak maximum principal stress, the C3D8I Finite Element (FE) provides good estimate of the peak stress even with the coarsest mesh. The quarter ring is meshed whit $2^{\star} 2 \mathrm{FE}$ in the cross-section corresponding to four integration points through the cross-section. The artery is meshed whit a hybrid fully-integrated linear hexahedron $\mathrm{FE}$ that is denoted in Abaqus by C3D8H. The cylinder is meshed with quadratic shell FE. The average FE length size of artery and plaque is $0.15 \mathrm{~mm}$. To sum up here is the following table 3 .

\begin{tabular}{|l|l|l|}
\hline Elements & Type of elements & Number of elements \\
\hline Stent & C3DR8I & 11682 \\
\hline Artery & C3DR81 & 26341 \\
\hline Expandable balloon & S4R & 4378 \\
\hline
\end{tabular}

Table 3: Mesh used.

\section{Deployment of the stent}

Finite element modelling of the stent deployment depends on the geometrical characteristics of the stent and the boundary conditions. In this context, two different geometries are used, as shown in Figure 1. The both configurations have the same dimensions: length of $4.70 \mathrm{~mm}$, an internal diameter of $1.30 \mathrm{~mm}$ and a thickness of $0.08 \mathrm{~mm}$. the difference between these geometries is the shape of the struts. Indeed, one is of the U-shape and the second of a $\Omega$-shape. The ends of the stent are maintained completely constrained in all degrees of freedom. This condition is supported by the fact that the patient remains immobile until the entire stabilization of the stent in the body. The loading consists in a radial displacement of the balloon, assimilated to a cylinder, of about $0.45 \mathrm{~mm}$. The radial displacement is exerted on the internal surface of the cylinder to expand the blood vessel. To avoid the difficulties of computation convergence, due to the nonlinear aspect induced by the boundary conditions, the contact interaction between both the cylinder and the stent and the stent and the artery should be carefully modelled. In this way, several formulations have been proposed in the literature to solve the contact problems [14]. The penalty method, which consists of the introduction of a penalised function, is one of them and makes it possible to transform the initial constrained problem into an unconstrained one. To prevent the chattering behavior, that might occur due to the differences in mass densities between the stent and the plaque materials during contact, the contact interaction is modelled with this method and the friction coefficient is chosen to be 0.1 . The simulation time lasts for $10^{-3} \mathrm{~s}$ and the time increment is fixed to $5 \times 10^{-8} \mathrm{~s}$.

The stent is loaded according to the Hollomon constitutive law $\sigma=\mathrm{k} \varepsilon^{\wedge} \mathrm{p}$, with $\mathrm{k}=1036.44 \mathrm{MPa}$ and $\mathrm{p}=0.16$, while the mechanical behavior of the artery is based on the isotropic, homogeneous and hyper-elastic model of Holzapfel et al. [15]. This model is found to thoroughly describe the nonlinear stress-strain relationship of the elastic arterial tissue. The constitutive model is based on a reduced strain energy density function of sixth order, such as:

$$
\begin{aligned}
& U=C_{1}\left(\dot{I}_{1}-3\right)+C_{2}\left(\dot{I}_{1}-3\right)^{2}+C_{3}\left(\dot{I}_{1}-3\right)^{3}+ \\
& C_{4}\left(\dot{I}_{1}-3\right)^{4}+C_{5}\left(\dot{I}_{1}-3\right)^{5}+C_{6}\left(\dot{I}_{1}-3\right)^{6}
\end{aligned}
$$

Where the values of the $\mathrm{C}_{\mathrm{i}}$ parameters are:

$$
\mathrm{C}_{1}=0.00652, \mathrm{C}_{2}=0.0489, \mathrm{C}_{3}=0.00926, \mathrm{C}_{4}=0.76, \mathrm{C}_{5}=-0.43, \mathrm{C}_{6}=0.0869 \text {, }
$$

and $\overline{\mathrm{I}}_{1}$ is the first invariant of the Cauchy-Green tensor.

The Figure 2 shows the deployment of the stent with a $0.45 \mathrm{~mm}$ opening diameter. We note that the stress intensity is localized in the rounded areas.

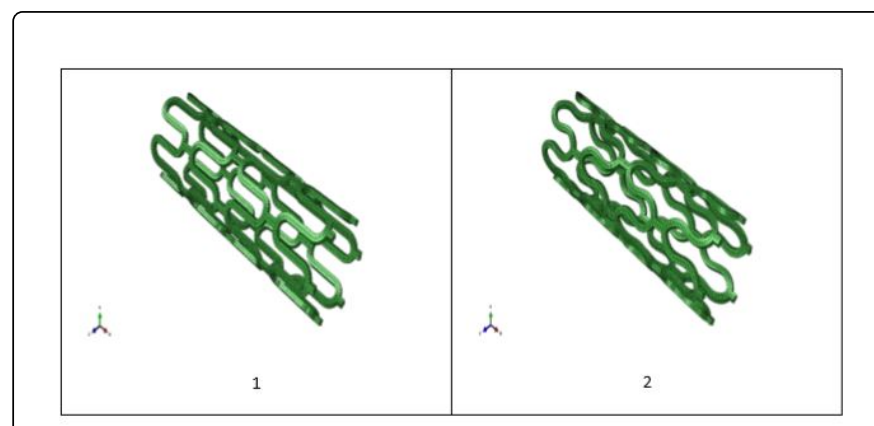

Figure 1: The geometries adopted for this study.

\section{Application of Bending loading}

The output database (.odb) file obtained after the expansion stage is imported in Abaqus standard to account for the residual stresses. Then, the bending is studied by applying a load on the external elements of the stent Figure 3. 


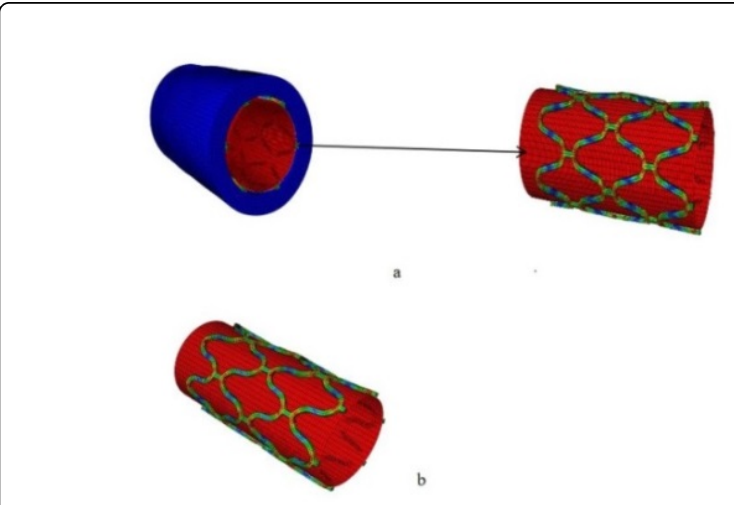

Figure 2: Deployement of the stent. A: U-shape. B: $\Omega$-shape.

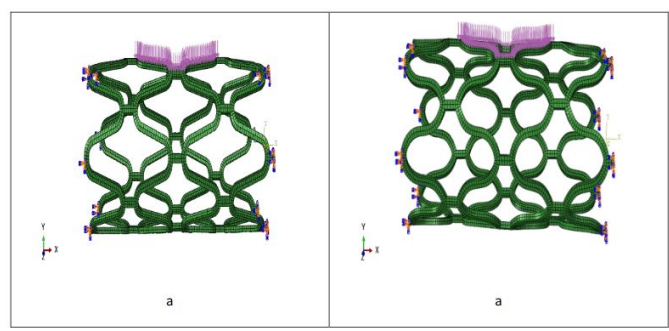

Figure 3: Application of bending loading. A: U-shape. B: $\Omega$-shape.

\section{The effect of bending loading on the stent durability}

Many numerical and clinical studies have shown that stent fracture after implantation causes serious cardiovascular complications. This fracture happens before the stent's lifetime predicted by the manufacturer. $78 \%$ of serious complications of the stent are caused by stent rupture [16]. However, several studies have shown that vascular movement has a significant impact on stent resistance. Umeda et al. [17], Shaikh et al. [18], Lee et al. [19] have shown that mechanical stresses such as bending, torsion, traction and compression produced by cardiac contractions causes very high stress concentrations. This concentration of stress is a major cause of stent rupture. Among the factors of increased stress there is flexion. The flexion of the coronary artery is caused by the cyclic movement of the artery and the formulation of restenosis [1].

\section{For U-shape}

There is an immediate diameter gain called acute gain after the implantation of a stent. But with time, the inflammatory response of the immunity system tends to push the stent back, hurting the vessel and losing a percentage of what was previously gained: this phenomenon is called « late loss».

The net gain in diameter is the difference between acute gain and late loss and is a measure of stent-graft effectiveness [20]. In addition to the retressissement there is a movement of the artery due to the bending of the stent, that leads to an early rupture that cause serious complications for the patient.
We modeled the bending using the Abaqus software we applied a pressure on the external elements of the stent while keeping its extremities totally constrained. The applied pressure is changed until getting the rupture that is $580 \mathrm{MPa}$ for an AISI $316 \mathrm{~L}$.

The results of Figure 4 shows the rupture produced by a pressure of $1,75 \mathrm{MPa}$. We also note that the contraint of Von Mises rises quickly as soon as we get over 0,5 MPa. We notice that the stress that causes the rupture is very weak; this can be justified by the plastification engendered by the applied shifting to realize the opening.

Moreover, the stress raiser is found to be localized in the round parts and connecting elements for the U-shape geometry. cf. Figure 5.

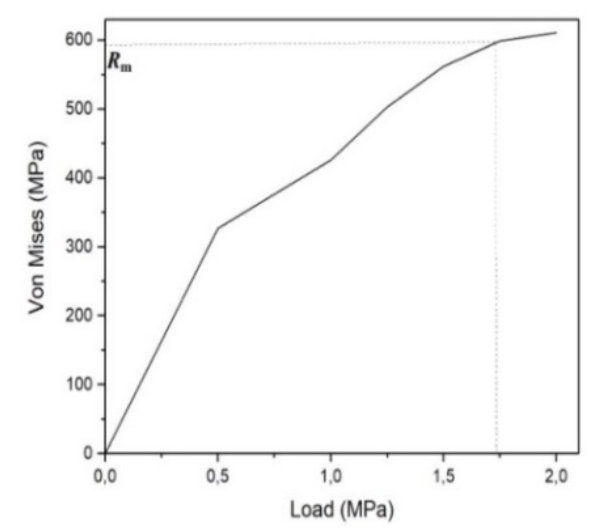

Figure 4: Von Mises stress as a function of the imposed load for Usahpe geometry.

\section{For $\Omega$-shape}

As previously stated the movement of the artery causes an increase in the stress intensity in some elements of the stent. This increase leads to serious complications such as stent rupture. The bending of the stent is one of the most serious complications because excessive bending leads to rupture.

In this context Figure 6 shows the evolution of the Von Mises stress in function of the imposed flexion. We note that the behavior of the $\Omega$ shape geometry is similar to the behavior of the U-shape geometry.

Indeed the Von Mises stress increases by increasing the bending until reaching the break at about $1.83 \mathrm{MPa}$. We also note that the geometry of $\Omega$-shape is more resistance compared to the U-shape's one.

This difference is observed in the loading of the necessary bending to obtain the rupture.

In the same way as the geometry of the U-shape, the location of the Von Mises stress is localized in the connection elements between the different strusts of $\Omega$-shape. This concentration of stress shows that these elements have most chances to break. cf. Figure 7. 


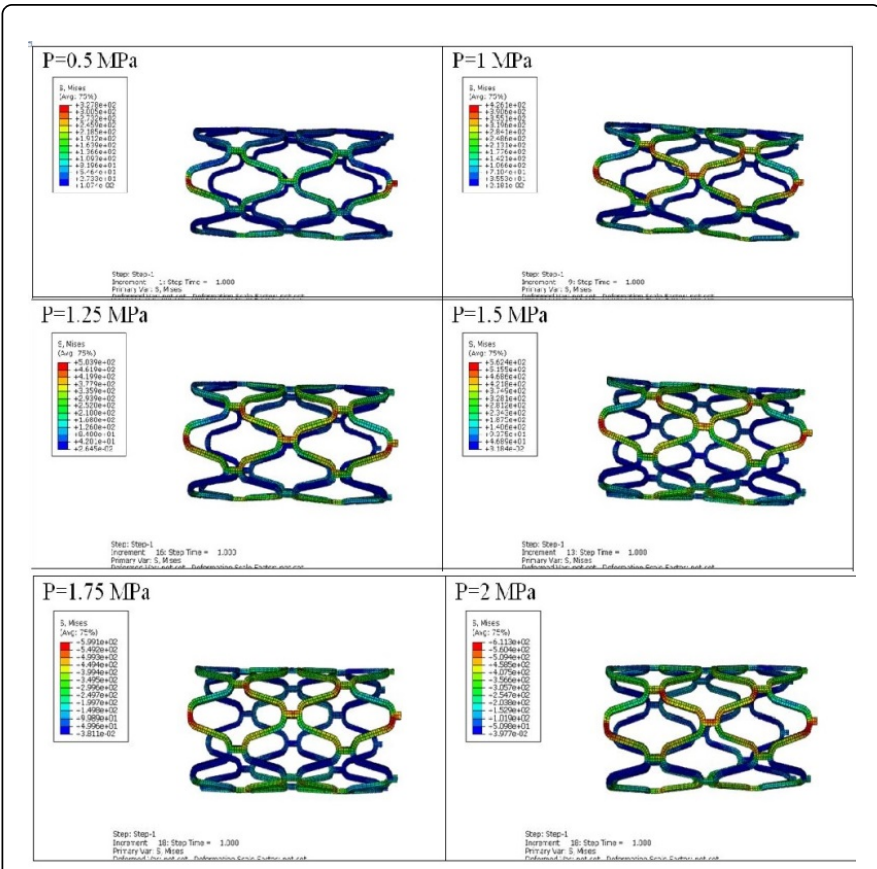

Figure 5: Stress raiser location for U-shape geometry.

\section{Comparison between two geometries}

It should be noted that the bending loading imposed on the geometry of $U$-shape is the same as the one imposed on the geometry of $\Omega$-shape. For a fair comparison, the cures corresponding to each case are represented in terms of Von Mises stress and gathered on the same graph to observe the resistance of each geometries.

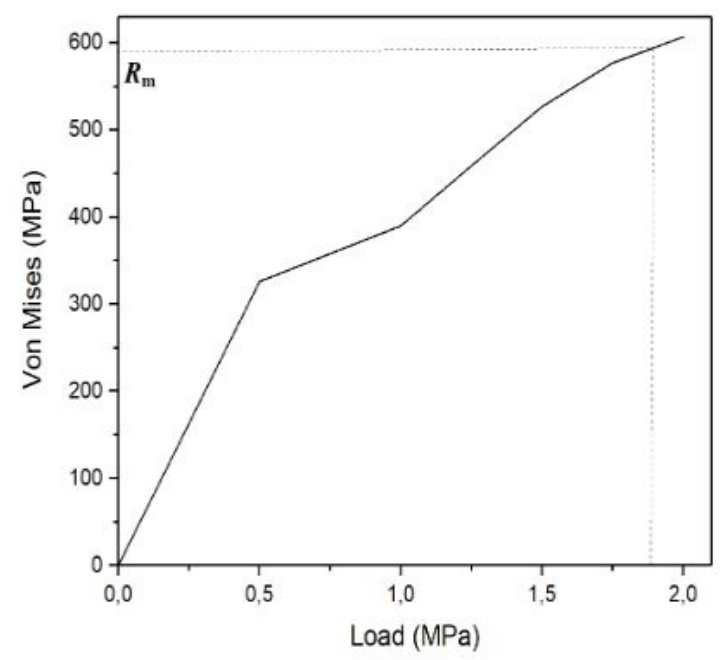

Figure 6: Von Mises stress as a function of the imposed load for $\Omega$ sahpe geometry.

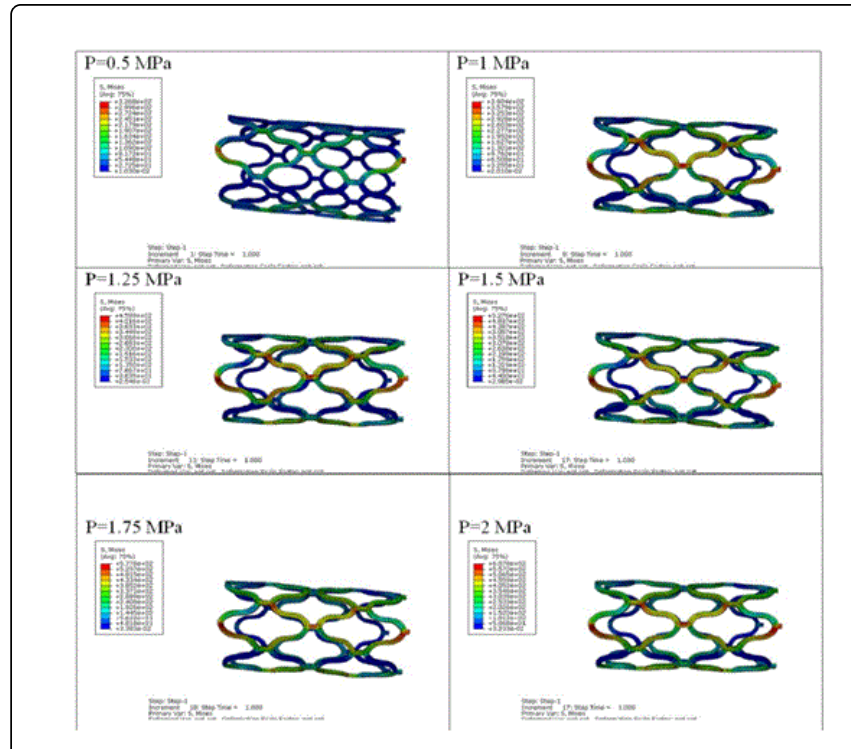

Figure 7: Stress raiser location for $\Omega$-shape geometry.

Figure 8 clearly shows that the geometry of $\Omega$-shape is more resistant, for the same bending loading, than the geometry of U-shape. This behavior may be associated with the broad distribution of the stress and strain on the stent of $\Omega$-shape.

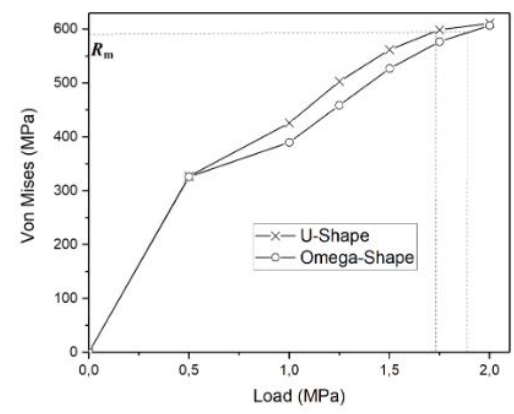

Figure 8: Resistance comparison between the two geometries studied.

\section{Conclusion}

In this study, the finite element method has been used to analyze the effect of the bending loading on the durability of two geometries of stent made of AISI 316L. Many studies have shown that the stent undergoes high fatigue cycle when subjected to blood pressure. However, it is found that the stent sometimes experiences in-service failure. Among the causes of stent rupture is the bending caused by the movement of the artery? Indeed, when the stent is loaded in flexion the rupture is reached faster and for rather low pressures. U-shape geometry the fracture occurs for a bending load of 1.75 MPa. While for the $\Omega$-shape geometry the break occurs for a load of $1.83 \mathrm{MPa}$. In addition, the evolution of the stress of Von Mises evolves quickly with the loading increase of the bending. Moreover, there is a difference of resistance between the two geometries. Indeed the geometry of $\Omega$ - 
shape is more resistant compared to the U-shape. This result shows that the forme of geometry plays an important role in the durability of the stents which explains the large number of geometries with different design.

\section{References}

1. Benhaddou M, Abbadi M, Ghammouri M (2016) Numerical modeling of AISI 316L cardiovascular stent behavior under blood pressure and restenosis loadings. J Biomim Biomater Biomed Eng 27: 60-76.

2. Mahnken AH (2012) CT imaging of coronary stents: Past, present, and future. ISRN Cardiol pp: 1-12.

3. Bennett MR (2003) In-stent stenosis: Pathology and implications for the development of drug eluting stents. Heart 89: 218-224.

4. Auricchio F, Constantinescu A, Scalet G (2014) Fatigue of 316L stainless steel notched lm-size components. Int J Fatigue 68: 231-247.

5. Sweeney CA, McHugh PE, McGarry JP, Leen SB (2012) Micromechanical methodology for fatigue in cardiovascular stents. Int $\mathrm{J}$ Fatigue 44: 202-216.

6. Philippe F, Dibie A, Larrazet F, Meziane T, Folliguet T, et al. (2005) Drug eluting stents: from evidence based medicine to clinicalpractice. Ann Cardiol Angeiol 54: 201-211.

7. Shaikh F, Maddikunta R, Djelmami-Hani M, Solis J, Allaqaband S, et al. (2008) Stent fracture, an incidental finding or a significant marker of clinical in-stent restenosis? Catheter Cardiovasc Interv 71: 614-618.

8. Sianos G, Hofma S, Ligthart JMR, Saia F, Hoye A, et al. (2004) Stent fracture and restenosis in the drug-eluting stent era. Catheter Cardiovasc Interv 61: 111-116.

9. Gervaso F, Capelli C, Petrini L, Lattanzio S, Di Virgilio L, et al. (2008) On the effects of different strategies in modelling balloon-expandable stenting by means of finite element method. J Biomech 41: 1206-1212.

10. Azaouzi M, Makradi A, Petit J, Belouettar S, Polit O (2013) On the numerical investigation of cardiovascular balloon-expandable stent using finite element method. Comp Mat Sci 79: 326-335.
11. Matthys KS, Alastruey J, Peiro J, Khir AW, Segers P, et al. (2007) Pulse wave propagation in a model human arterial network: Assessment of 1-D numerical simulations against in vitro measurements. J Biomech 40: 3476-3486.

12. Costa MA, Sabate M, Kay IP, Feyter P, (2000) Three-dimensional intravascular ultrasonic volumetric quantification of stent recoil and neointimal formation of two new generation tubular stents. Am J Cardiol 85: 135-139.

13. Schievano S, Parenzan G, Migliavacca F, Petrini L, Dubini G, et al. (2006) Stent fracture in percutaneous pulmonary valve implantation: a finite element study. J Biomech 39: 292-293.

14. Abichou H, Zahrouni H, Potier-Ferry M (2002) Asymptotic numerical method for problems coupling several nonlinearities. Comput Methods Appl Mech Engrg 191: 5795-5810.

15. Holzapfel GA, Sommer G, Gasser CT, Regitnig P (2005) Determination of layer-specific mechanical properties of human coronary arteries with no atherosclerotic intimal thickening and related constitutive modeling. Am J Physiol Heart Circ Physiol 289: 2048-2058.

16. Nakazawa G, Finn AV, Vorpahl M , Ladich E, Kutys R, et al (2009). Incidence and predictors of drugeluting stent fracture in human coronary artery: a pathologic analysis. J Am Coll Cardiol 54: 1924-1931.

17. Umeda H, Gochi T, Iwase M, Izawa H, Shimizu T, et al. (2009). Frequency predictors and outcome of stent fracture after sirolimus-eluting stent implantation. Int J Cardiol 133: 321-326.

18. Shaikh F, Maddikunta R, Djelmami-Hani M, Solis J, Allaqaband S, et al. (2008) Stent fracture, an incidental finding or a significant marker of clinical in-stent restenosis? Catheter Cardiovasc Interv 71: 614-618.

19. Lee MS, Jurewitz D, Aragon J, Forrester J, Makkar RR, Kar S. Stent fracture associated with drug-eluting stents: clinical characteristics and implications. Catheter Cardiovasc Interv 69: 387-394.

20. Dangas GD, Claessen BE, Caixeta A, Sanidas EA, Mintz GS, et al. (2010) In-stent restenosis in the drug-eluting stent era. J American College Cardiol 56: 1897-1907. 